\title{
Perinephric Pseudocyst in a Two-Month-Old Female Cat
}

\author{
Gabriela da Cruz Schaefer', Viviana Cauduro Matescoㄹ, Paula Reis Pereira', Welden Panziera', \\ David Driemeier ${ }^{3}$, Saulo Petinatti Pavarini ${ }^{3}$ \& Fernanda Vieira Amorim da Costa ${ }^{4}$
}

\begin{abstract}
Background: Perinephric pseudocyst is defined as an accumulation of fluid in fibrous sacs surrounding one or both kidneys, and may be located in the subcapsular or extracapsular region. Histologically, it is characterized by the absence of an epithelial lining to the cyst wall and the term pseudocyst is used. This condition have been reported sporadically in cats, and is associated with chronic kidney disease, mainly in older animals. The diagnosis is based on imaging tests and the prognosis is related to the severity of renal dysfunction. The aim of this report is to describe an unusual presentation of perinephric pseudocyst in a very young female cat.

Case: A mixed-breed 2-month-old female cat was evaluated for presenting abdominal distension and anatomical deformities of the limbs. Renomegaly on the left side was noted during abdominal palpation. On presentation, the serum creatinine was $134 \mu \mathrm{mol} / \mathrm{L}$. Urinalysis showed mild proteinuria, presence of squamous and transitional cells, and rare bacteria. The urine specific gravity was 1.044 and urine protein to creatinine ratio was 0.23 . Abdominal ultrasonography revealed a large anechoic subcapsular cyst on the left kidney, in adittion to dilatation of renal pelvis and calyces. The excretory urography showed left renomegaly, and the nephrogram and pyelogram were not clearly observed in this kidney, indicating unilateral deficiency in renal filtration. The cat was referred to unilateral left nephrectomy but died shortly before surgery. At necropsy, marked left kidney enlargment was observed $(6.5 \times 4.5 \times 1.5 \mathrm{~cm})$, with a cystic aspect, occupying almost the entire abdominal cavity. The cystic content was translucent and was compressing the renal parenchyma, resulting in hydronephrosis. The capsule forming the cyst wall was composed of connective tissue, characterizing the perinephric pseudocyst. Discussion: Reports show cases of perinephric pseudocyst affecting mostly older animals, above eight years old. In one retrospective study of 26 cases, $73 \%$ were male and the mean age was eleven years, ranging from four to 18 years old. Another study of 13 cases showed a mean age of 16 years. In this report, unexpectedly, the patient was too young compared to these other cases, but the clinical presentation was similiar. Being a young animal, in the absence of ureterolithiasis or history of trauma, it is suggested a congenital origin for the disease. Loss of renal function and azotemia may occur in about $90 \%$ of the cases by compression of the renal parenchyma by the pseudocyst or due to associated interstitial fibrosis. In this case, the serum creatinine concentration was above the reference value proposed for pediatric patients therefore, the cat may be considered azotemic. Beyond that, the excretory urography indicated no proper excretory function on the left kidney. It is proposed that the renal function was being compensated by the contralateral kidney. The clinical and imaging findings of this case are compatible with perinephric pseudocyst, which was confirmed by necropsy and histopathological examination. This condition is not commonly reported in cats of pediatric age, althought it must be considered as a differencial diagnosis to cats presenting abdominal distension and renomegaly, even young ones. Imaging tests were important in this case, not only to direct the diagnosis, but also contributing in evaluating the renal excretory function.
\end{abstract}

Keywords: perirenal pseudocyst, pararenal cyst, feline.

${ }^{1}$ Programa de Pós-graduação em Ciências Veterinárias (PPGCV), Universidade Federal do Rio Grande do Sul (UFRGS), Porto Alegre, RS, Brazil. ${ }^{2}$ M.V. do Hospital Veterinário Uniritter, Porto Alegre, RS. ${ }^{3}$ Setor de Patologia Veterinária, Departamento de Patologia Clínica Veterinária (DPCV) \& ${ }^{4}$ Serviço de Medicina Felina, Departamento de Medicina Animal (DMA), Faculdade de Veterinária (FaVet), UFRGS, Porto Alegre. CORRESPONDENCE: G.C. Schaefer [gabrielacschaefer@gmail.com - Tel.: +55 (51) 3308 6095]. Hospital de Clínicas Veterinárias - UFRGS. Av. Bento Gonçalves n. 9090. Bairro Agronomia. CEP 91540-000 Porto Alegre, RS, Brazil. 


\section{INTRODUCTION}

Perinephric pseudocyst consists of an accumulation of fluid in fibrous sacs surrounding one or both kidneys, and may be located in the subcapsular or extracapsular region [2]. Histologically, it is characterized by the absence of an epithelial lining to the cyst wall and the term pseudocyst is used. The etiology of this condition includes accumulation of transudate due to increased hydrostatic pressure, lymphatic obstruction or rupture of renal cysts; accumulation of hemorrhagic fluid due to trauma, tumors, coagulation disorders and vascular accidents; or accumulation of urine due to rupture of renal pelvis or ureter, congenital defects of the urinary tract or obstructive processes [6].

This condition have been reported sporadically in older cats associated with chronic kidney disease, which can block venous drainage due to fibrosis and contraction of renal parenchyma [2]. There is no breed predisposition and male cats are more often affected $[2,8]$. Clinical presentation includes abdominal distention, polyuria, polydpsia, weight loss, anorexia and vomiting [2].

The presumptive diagnosis is made by imaging tests, mainly abdominal ultrasonography, which shows an anechoic fluid between the capsule and the renal parenchyma [3]. Excretory urography can evaluate the size and shape of the kidneys, as well as the relative function of the organ [4]. Because the structure does not communicate with renal parenchyma, contrast does not fill it [8]. Surgical resection of cyst wall is recommended and the prognosis is related to the severity of renal dysfunction [8]. The aim of this report is to describe an unusual presentation of perinephric pseudocyst, in a two-month-old female cat.

\section{CASE}

A mixed-breed 2-month-old female cat was evaluated, in the Veterinary Teaching Hospital of Federal University of Rio Grande do Sul, for presenting abdominal distension and anatomical deformities of the limbs. Medical history was unknown since the animal had been rescued from the street a few days ago. Renomegaly on the left side was noted by abdominal palpation. On presentation, complete blood count was within reference range and the serum creatinine was $134 \mu \mathrm{mol} / \mathrm{L}$. Immunochromatographic test for FIV and FeLV (SNAP ${ }^{\circledR}$ FIV/FeLV Combo Test $)^{1}$ was negative. Urinalysis showed mild proteinuria and the presence of squamous and transitional cells, and rare bacteria. The urine specific gravity was 1.044 and the urine protein to creatinine ratio was 0.23 . Abdominal ultrasonography revealed a large anechoic subcapsular cyst on the left kidney, in adittion to dilatation of renal pelvis and calyces. The excretory urography showed left renomegaly, and the nephrogram and pyelogram were not clearly observed in this kidney, indicating unilateral deficiency in renal filtration.

The cat was referred to unilateral left nephrectomy but died shortly before surgery. At necropsy marked left kidney enlargment was observed $(6.5 \times 4.5 \times 1.5 \mathrm{~cm})$, with a cystic aspect, occupying almost the entire abdominal cavity (Figure 1A). The cystic content was translucent and was compressing the renal parenchyma, resulting in hydronephrosis (Figure 1B). In the histopathological examination, there was a marked compression of the left kidney and a decrease in its parenchyma, with a discrete multifocal lymphocytic infiltrate. The capsule forming the cyst wall was composed of connective tissue. Macrophages and rare neutrophils were infiltrated on the cyst inner surface. The lack of an epitelial lining forming the cyst wall characterized the structure as an unilateral perinephric pseudocyst.
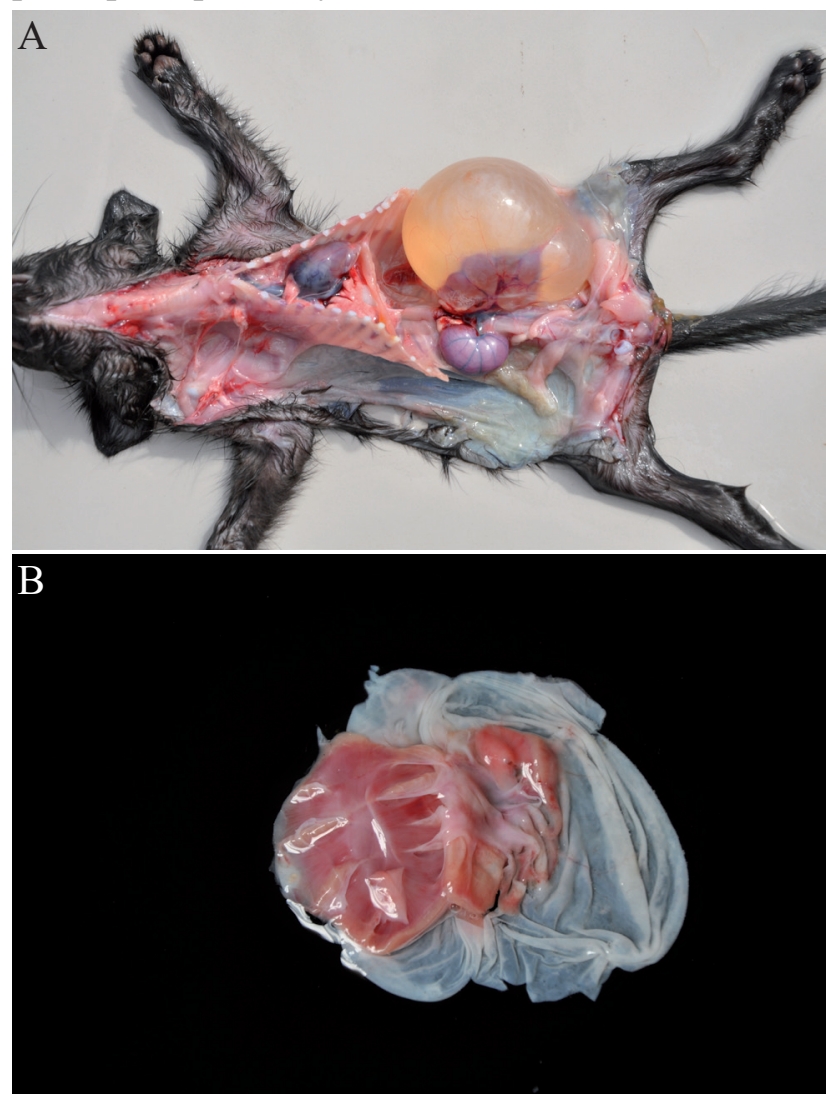

Figure 1. A- Left kidney markedly enlarged, of cystic aspect, occupying almost the entire abdominal cavity. B- Left kidney: hydronephrosis. 


\section{DISCUSSION}

Reports show cases of perinephric pseudocyst affecting mostly older animals, above eight years old $[6,9]$. In one retrospective study that included 26 cats with this condition, $73 \%$ were male and the mean age was eleven years, ranging from four to 18 years old. The main clinical sign presented was abdominal distension and most of the animals had some degree of renal dysfunction [1]. In another study with 13 cats, the mean age was 16 years old [10]. In this case report, unexpectedly, the patient was very young and female, but the clinical presentation was similar. In addition, for being a young animal in the abscence of ureterolithiasis or history of trauma, it is suggested a congenital origin for the disease.

Loss of renal function and azotemia may occur by compression of the renal parenchyma by the pseudocyst or due to associated interstitial fibrosis [8]. About $90 \%$ of cats have some degree of renal disease at the time of diagnosis [2]. In this case, the patient had serum creatinine within the reference value proposed by the International Renal Interest Society (up to 140 $\mu \mathrm{mol} / \mathrm{L})[5]$ and urine specific gravity was considered adequate. However, creatinine was at the upper limit and it is known that this is not an early marker of renal function. In addition, in cats at eight weeks of age the adjusted creatinine reference value is up to $106 \mu \mathrm{mol} / \mathrm{L}$ [7]. Therefore, considering this reference interval, the cat may be considered azotemic. Beyond that, the excretory urography indicated no proper excretory function on the left kidney, since no left nephrogram and pyelogram were observed. It is proposed that azotemia was not as severe due to the compensation of renal function by the contralateral kidney.

The clinical and imaging findings of this case are compatible with perinephric pseudocyst, which was confirmed by necropsy and histopathological examination. This condition is not commonly reported in cats of pediatric age, but should be considered as a differential diagnosis of patients presenting with abdominal distention and renomegaly, even when young. Imaging tests were important in this case, not only to direct the diagnosis, but also contributing in evaluating the renal excretory function.

\section{MANUFACTURER}

${ }^{1}$ IDEXX Laboratories Inc. Westbrook, ME, USA.

Declaration of interest. The authors report no conflicts of interest. The authors alone are responsible for the content and writing of the paper.

\section{REFERENCES}

1 Beck J.A., Bellenger C.R., Lamb W.A., Churcher R.K., Hunt G.B., Nicoll R.G. \& Malik R. 2000. Perirrenal pseudocysts in 26 cats. Australian Veterinary Journal. 78(3): 166-171.

2 Da Costa F.V.A. 2011. Perinephric Pseudocysts. In: Norsworhty G.D., Grace S.F., Crystal M.A. \& Tilley L.P. (Eds). The Feline Patient. 4th edn. Ames: Blackwell Publishing, pp.394-396.

3 Debruyn K., Haers H., Combes A., Paepe D., Peremans K., Vanderperren K. \& Saunders J.H. 2012. Ultrasonography of the feline kidney: Technique, anatomy and changes associated with disease. Journal of Feline Medicine and Surgery. 14: 794-803.

4 Heuter K.J. 2005. Excretory Urography. Clinical Techniques in Small Animal Practice. 20: 39-45.

5 IRIS (Internacional Renal Interest Society). 2016. IRIS Staging of CKD (modified 2015). Fonte: <http://www.iriskidney.com/pdf/staging-of-ckd.pdf>. [Accessed online in April 2017].

6 Lemire T.D. \& Read W.K. 1998. Macroscopic and Microscopic Characterization of a Uriniferus Perirenal Pseudocyst in a Domestic Short Hair Cat. Veterinary Pathology. 35: 68-70.

7 Levy J.K., Crawford C. \& Werner L.L. 2006. Effect of age on reference intervals of serum biochemical values in kittens. Journal of the American Veterinary Medical Association. 228(7): 1033-1037.

8 Scherk M. 2012. Urinary Tract Disorders: The upper urinary tract. In: Little S.E. (Ed). The Cat: Clinical Medicine and Management. St Louis: Elsevier Saunders, pp.935-980.

9 McCord K., Steyn P.F. \& Lunn K.F. 2008. Unilateral improvement in glomerular filtration rate after permanent drainage of a perinephric pseudocyst in a cat. Journal of Feline Medicine and Surgery. 10: 280-283.

10 Ochoa V.B., DiBartola S.P., Chew D.J., Westropp J., Carothers M. \& Biller D. 1999. Perinephric pseudocysts in the cat: a retrospective study and review of the literature. Journal of Veterinary Internal Medicine. 13(1): 47-55. 